\title{
TOWARDS A LARGE-SCALE ASSESSMENT OF THE RELATIONSHIP BETWEEN BIOLOGICAL AND CHRONOLOGICAL AGING: THE INSPIRE MOUSE COHORT
}

\author{
Y. SANTIN ${ }^{1}$, S. LOPEZ ${ }^{2}$, I. ADER ${ }^{5}$, S. ANDRIEU ${ }^{3,4}$, N. BLANCHARD ${ }^{6}$, A. CARRIÈRE ${ }^{5}$, L. CASTEILLA ${ }^{5}$, \\ B. COUSIN ${ }^{5}$, N. DAVEZAC ${ }^{2}$, P. DE SOUTO BARRETO ${ }^{3,4}$, C. DRAY ${ }^{1}$, N. FAZILLEAU ${ }^{6}$, \\ D. GONZALEZ-DUNIA ${ }^{6}$, P. GOURDY ${ }^{1}$, S. GUYONNET ${ }^{3,4}$, N. JABRANE-FERRAT ${ }^{6}$, O. KUNDUZOVA ${ }^{1}$, \\ F. LEZOUALC' ${ }^{1}{ }^{1}$, R. LIBLAU ${ }^{6}$, L.O. MARTINEZ ${ }^{1}$, C. MORO ${ }^{1}$, P. PAYOUX $^{7}$, L. PÉNICAUD $^{5}$, \\ V. PLANAT-BÉNARD ${ }^{5}$, C. RAMPON ${ }^{2}$, Y. ROLLAND ${ }^{3,4}$, J.-P. SCHANSTRA ${ }^{1}$, F. SIERRA ${ }^{9}$, P. VALET $^{1}$, \\ A. VARIN ${ }^{5}$, N. VERGNOLLE ${ }^{8}$, B. VELLAS ${ }^{3,4}$, J. VIÑA $^{10}$, B.P. GUIARD ${ }^{2}$, A. PARINI ${ }^{1}$ \\ 1. Institut des Maladies Métaboliques et Cardiovasculaires, Inserm, Université Paul Sabatier, UMR 1048 - I2MC, Toulouse, France; 2. Centre de Recherches sur la Cognition Animale \\ (CRCA), Centre de Biologie Intégrative (CBI), Université de Toulouse, CNRS, UPS, Toulouse, France; 3. Gerontopole of Toulouse, Institute of Ageing, Toulouse University Hospital \\ (CHU Toulouse), Toulouse, France; 4. UPS/Inserm UMR1027, University of Toulouse III, Toulouse, France; 5. STROMALab, CNRS ERL 5311, Etablissement Français du Sang- \\ Occitanie (EFS), National Veterinary School of Toulouse (ENVT), Inserm U1031, University Toulouse III Paul Sabatier, Toulouse, France; 6. Centre de Physiopathologie Toulouse \\ Purpan, INSERM/CNRS/UPS UMR 1043, University of Toulouse III, Toulouse, France; 7. ToNIC, Toulouse NeuroImaging Center, Université de Toulouse, Inserm, UPS, France; \\ 8. IRSD, Université de Toulouse, INSERM, INRA, ENVT, UPS, U1220, CHU Purpan, CS60039, 31024, Toulouse, France; 9. Division of Aging Biology, National Institute on Aging

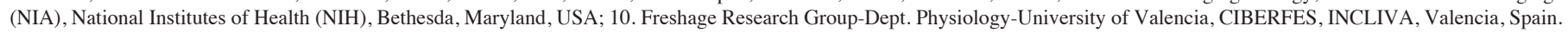

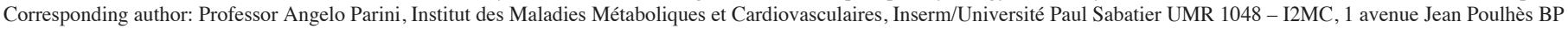 \\ 8422531432 Toulouse Cedex 4 - France, Phone: (+33)561325601, e-mail: angelo.parini@inserm.fr
}

\begin{abstract}
Aging is the major risk factor for the development of chronic diseases. After decades of research focused on extending lifespan, current efforts seek primarily to promote healthy aging. Recent advances suggest that biological processes linked to aging are more reliable than chronological age to account for an individual's functional status, i.e. frail or robust. It is becoming increasingly apparent that biological aging may be detectable as a progressive loss of resilience much earlier than the appearance of clinical signs of frailty. In this context, the INSPIRE program was built to identify the mechanisms of accelerated aging and the early biological signs predicting frailty and pathological aging. To address this issue, we designed a cohort of outbred SWISS mice (1576 male and female mice) in which we will continuously monitor spontaneous and voluntary physical activity from 6 to 24 months of age under either normal or high fat/high sucrose diet. At different age points $(6,12,18$, 24 months), multiorgan functional phenotyping will be carried out to identify early signs of organ dysfunction and generate a large biological fluids/feces/organs biobank (100,000 samples). A comprehensive correlation between functional and biological phenotypes will be assessed to determine: 1) the early signs of biological aging and their relationship with chronological age; 2) the role of dietary and exercise interventions on accelerating or decelerating the rate of biological aging; and 3) novel targets for the promotion of healthy aging. All the functional and omics data, as well as the biobank generated in the framework of the INSPIRE cohort will be available to the aging scientific community. The present article describes the scientific background and the strategies employed for the design of the INSPIRE Mouse cohort.
\end{abstract}

Key words: INSPIRE program, biological aging, mouse cohort, frailty, biomarkers.

\section{Introduction}

The improvement of medical care and living conditions has increased life expectancy. Although being a progress per se, the extension of life expectancy is associated with an elevated risk of all types of chronic diseases as well as the decline in intrinsic capacities (1). Research on the basic "biology of aging" aims to increase life expectancy and to improve the quality of life. In this context, geroscience has emerged as a new interdisciplinary field seeking to define the biological underpinnings of aging that lie at the crossroads of age-dependent biology, chronic disease and health $(2,3)$. The geroscience hypothesis postulates that, since aging plays a major role in most chronic diseases, addressing aging physiology will reduce or delay the onset of multiple age-associated defects.

Frailty is a clinical state of increased vulnerability resulting from aging-related decline in function and reserve across multiple physiological systems, that carries an increased risk for poor health outcomes including falls, incident disability, hospitalization, and mortality (4). Even though frailty is an age-associated syndrome, the idea that it is not a normal and inevitable part of aging is growing. Hence, frailty can be conceptualized as a result of accelerated biological aging (5), and elucidating its etiology is thus critical for its prevention and/or treatment. Therefore, there is a pressing need to discover markers to differentiate biological age from chronological age 
Figure 1

Parallel between INSPIRE Mouse and Human cohorts

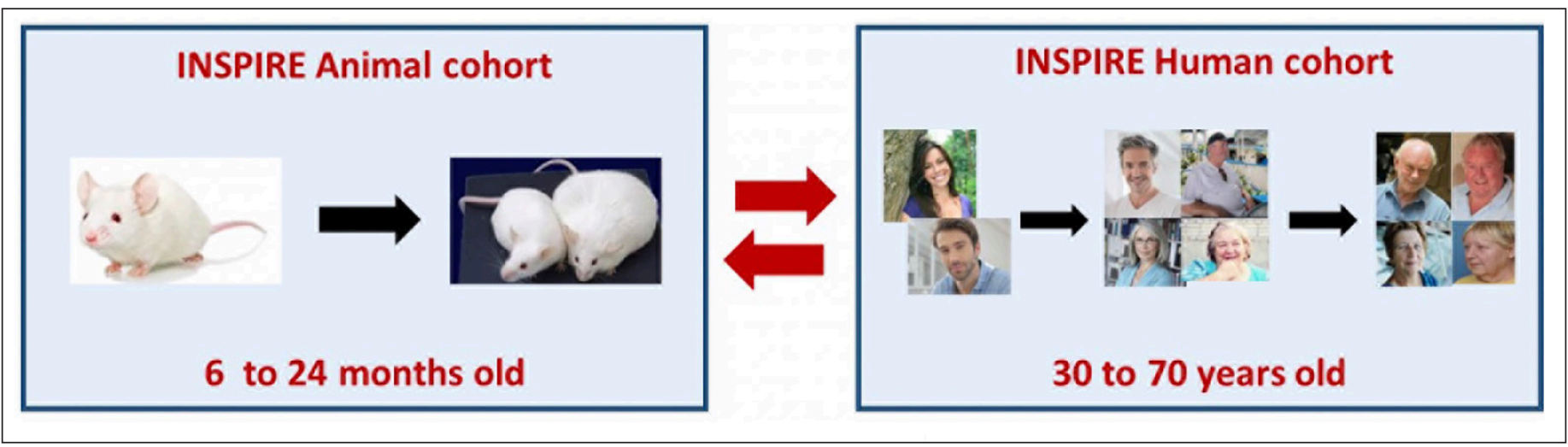

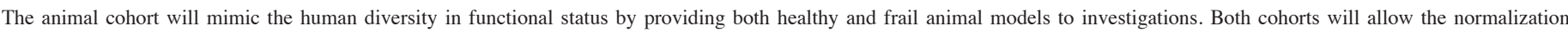

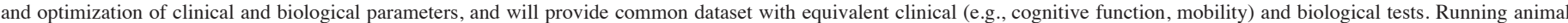

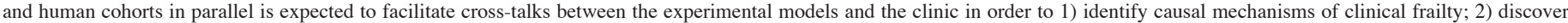

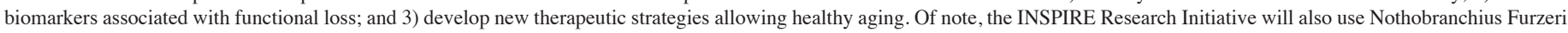
(African Killifish) and pet dogs as additional cohorts to investigate aging process.

and to identify individuals at higher risk of developing chronic diseases, ultimately with the goal to propose pharmacological and non-pharmacological approaches targeting biological processes underlying aging.

According to this integrated view, the INSPIRE research program has been created to foster research in the field of geroscience and healthy aging. INSPIRE aims at promoting healthy aging and preventing dependency through, among other strategies, the constitution of a bio-resource platform going from animals to humans in order to provide clinical, biological and technological resources for research and development on aging (for a detailed review on the INSPIRE program, see (6)). Besides the implementation of digital medicine (ICOPE program from the WHO) and the constitution of an INSPIRE Human Translational Cohort $(6,7)$, the INSPIRE program will create a unique Mouse Cohort dedicated to basic research, whose setup and design will be described in the present article.

\section{Overview of the INSPIRE Mouse cohort}

The primary goal of the INSPIRE Mouse cohort is to foster an understanding of the close relationship between the molecular mechanisms of biological aging and the onset of clinical frailty. This approach will importantly lead to the identification of frailty biomarkers. Complementarily, the INSPIRE Mouse cohort will enable to better characterize frailty in mice by implementing already existing tools such as the "Valencia Score", a frailty score mainly based on neuromuscular alterations (8), or the "Howlett and Rockwood frailty index" relying on a list of deficits that accumulate during aging (9). This will eventually lead to the creation of an "INSPIRE Frailty Score" suitable for mice and as close as possible to the clinical scenario in humans. Since multiple molecular pathways are involved in the aging process and can contribute to various aspects of frailty, a panel of valid biomarkers in combination with functional measures of frailty would allow both diagnosis and follow up in preclinical and clinical settings (10).

A major asset of the INSPIRE Mouse cohort is its "mirroring" of the INSPIRE Human cohort in order to facilitate the translation of results from basic science to humans (Figure 1). To further improve the extrapolation of the results to the clinic, "humanized" living conditions, i.e. high fat/high sucrose diet and sedentary lifestyle, will be studied as common risk factors of accelerated aging. A particular attention has also been paid to the selection of a mouse strain that congruently mimics human heterogeneity. Besides, as main studies on frailty have been done on either male or female mice, comparison of frailty between genders will also be a major advantage of the INSPIRE Mouse cohort. These important considerations should facilitate the crosstalk between humans and experimental models, therefore speeding up the discovery process (Figure 1).

Here, we provide detailed information on the INSPIRE Mouse cohort setup, by putting forward an innovative methodology ranging from the study design to comprehensive phenotyping. This will be done by integrating measures evaluating different dimensions of frailty including cognitive/ motor capacities, cardiac function assessment, body composition, metabolic parameters, urinary incontinency and immune function as defined in humans (syndrome diagnosis). Importantly, tissue biobanking for frailty biomarkers identification is implemented.

\section{Study design}

The INSPIRE Mouse cohort was designed to be as close as possible to human lifestyle. As a major issue in aging studies in mice is that most are carried out in inbred strains, the INSPIRE 


\section{THE JOURNAL OF FRAILTY \& AGING}

Mouse cohort will gather a genetically heterogeneous mouse stock to better mimic human diversity. In addition, besides normal aging, physical activity/exercise we will be studied as a human-relevant paradigm of delayed aging, while obesity/ overweight will be evaluated as a risk factor for accelerated aging in mice. These are well-known risk factors for frailty in humans (11-14) and, as compared to other experimental approaches, they are particularly suitable to promote cognitive $(15,16)$, cardiometabolic $(17,18)$ and immune dysfunctions (19), that are involved in progressive/long-term frailty $(20,21)$. These aspects are described below.

\section{Selection of mouse strain}

Animal models have been critical tools in biomedical research, and among them, the laboratory mouse is undoubtedly the most commonly used experimental non-human model. The prevalence of mouse models in biomedical research, in particular in the field of aging, is unsurprisingly considerable given that mice require relatively inexpensive care, reproduce quickly, and have a high genetic similarity to humans (22). Especially, inbred strains (like C57Bl/6J mice or BALB/c mice), transgenic and congenic mice with inbred backgrounds are most used. An inbred strain is defined as a strain that has been through at least 20 generations of sib-mating, making animals from the same inbred strain effectively genetically identical (i.e. isogenic) (23). However, such strains do not reflect genetically diverse human populations, and therefore constitute only a small part of the picture. Hence, outbred stocks (as contrary to inbred mice that are referred as strains, outbred mice are referred to as stocks) represent new research options that parallel or even exceed human genetic diversity, offering more generalizability of responses across populations. Unfortunately, unfamiliarity with outbred mice and concerns about difficulty, genetic variability and lack of reproducibility have impeded their widespread use by the research community. Nevertheless, while there is a common belief suggesting that inbred strains should present less variability of outcomes (24), presenting practical and ethical advantages, a recent review of the literature shows this to be erroneous. Indeed, several studies have shown that for a majority of readouts, inbred and outbred mice showed comparable phenotypic variations (25-27). In addition, Tuttle et al. performed a systematic review of the primary literature, and found that strain type (i.e. inbred or outbred) did not have any effect on within-strain variability regardless of trait category including anatomy, behavior, immune function, molecules and organ function (26). Therefore, except in cases where precise genotypic regulation or standardization is required, it appears that outbred stocks from heterogeneous backgrounds are more appropriate models in many biomedical research applications.

Among the available outbred stocks, the SWISS mice are commonly used. The initial stock was bred at the Centre Anti-Cancéreux Romand in Lausanne, Switzerland, in the 1920s and consisted of two male and seven female albino mice derived from a non-inbred stock. These mice have many advantages for long-term studies, as they are inexpensive, robust and commercially available. They have been used for mouse transgenesis experiments, principally due to efficient breeding and large litter sizes. Importantly, they have a large genetic diversity, which is similar to that found within and between human populations (28). In addition, SWISS mice are sensitive to high fat diets (29-33) and have been used in aging studies $(34,35)$, the latter being of primary importance for the INSPIRE project. Indeed, Antoch and collaborators reported that mean life expectancy of these mice was $121.1 \pm 9.2$ weeks for males and $109.6 \pm 6.9$ weeks for females, with maximum lifespan being of 150 weeks and 164 weeks respectively (35).

For the reasons stated above, the INSPIRE Mouse cohort will gather SWISS mice as a model mimicking the genetic heterogeneity of human populations. It is important to note that females are often underrepresented in animal studies, leading to a compromised understanding of female biology and resulting in poorer treatment outcomes for women. By looking primarily in males, important biological effects can be missed or misinterpreted, partly due to hormonal and genetic intrinsic differences. In addition, contrary to a common belief, recent analyses have found that variability in female performance without regard for the estrous phase is not higher than performance variability in males (36). We thus decided to include both male and female SWISS mice in the INSPIRE cohort in order to further improve the reliability and representativeness of our findings (Table 1). When planning a study that includes an advanced age group, it is important to provide extra animals to ensure sufficient statistical power as a result of early mortality. Therefore, the number of males and females in each group was statistically adjusted considering both spontaneous and high fat diet-induced mortality $(35,37)$ (Table 1). Finally, as the tracking of each mouse is critical to carry out an individual follow-up, microchips will be implanted in mice so they will be easily identified using a microchip reader.

\section{High fat high sucrose (HFHS) diet-induced obesity as a model of accelerated aging}

There is strong evidence that excessive adiposity contributes to the impairment of several parameters of frailty, notably reducing the ability of older adults to perform physical activities, impairing different forms of memory and increasing metabolic instability (38). Many obesity-related conditions including low-grade inflammation, insulin resistance, type 2 diabetes and low physical activity are risk factors for frailty. In order to study the biological and molecular changes that occur during aging, and to depict the differences between accelerated and normal aging, a model of diet-induced obesity will be used to induce accelerated aging.

At present, there is a range of commercial high-fat diets that have been demonstrated to make small rodents obese. However, some of these diets contain levels of dietary fat that are much 
Table 1

Mouse cohort organization

\begin{tabular}{|c|c|c|c|c|c|}
\hline \multirow{2}{*}{ Conditions } & \multicolumn{4}{|c|}{ Cross-sectional study } & \multirow[t]{2}{*}{ Longitudinal study } \\
\hline & 6 months & 12 months & 18 months & 24 months & \\
\hline Control & 80 mice $(40 \mathrm{M} / 40 \mathrm{~F})$ & 80 mice $(40 \mathrm{M} / 40 \mathrm{~F})$ & 100 mice $(52 \mathrm{M} / 48 \mathrm{~F})$ & 136 mice $(68 \mathrm{M} / 68 \mathrm{~F})$ & 120 mice $(60 \mathrm{M} / 60 \mathrm{~F})$ \\
\hline HFHS diet & -- & 80 mice $(40 \mathrm{M} / 40 \mathrm{~F})$ & 112 mice $(64 \mathrm{M} / 48 \mathrm{~F})$ & 180 mice $(112 \mathrm{M} / 68 \mathrm{~F})$ & \\
\hline $\begin{array}{l}\text { Voluntary Activity } \\
\text { (VA) }\end{array}$ & -- & 80 mice $(40 \mathrm{M} / 40 \mathrm{~F})$ & 100 mice $(52 \mathrm{M} / 48 \mathrm{~F})$ & 136 mice $(68 \mathrm{M} / 68 \mathrm{~F})$ & \\
\hline HFHS diet + VA & -- & 80 mice $(40 \mathrm{M} / 40 \mathrm{~F})$ & 112 mice $(64 \mathrm{M} / 48 \mathrm{~F})$ & 180 mice $(112 \mathrm{M} / 68 \mathrm{~F})$ & \\
\hline TOTAL & 80 mice & 320 mice & 424 mice & 632 mice & 120 mice \\
\hline
\end{tabular}

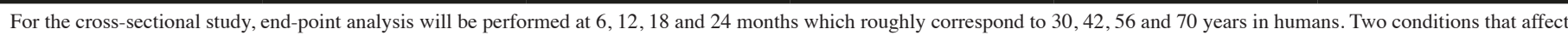

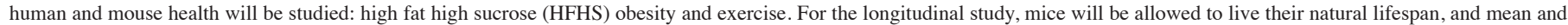
maximal lifespans will be then calculated. Both male (M) and female (F) SWISS mice will be included in the cohort.

higher than the levels that humans routinely consume. The typical American or European diet contains about 35-40\% fat by energy, and a tolerable high-fat human diet might contain $50-60 \%$ of energy as fat. However, the $60 \%$ fat rodent diet often used in experimental paradigms presents a much greater distortion of the fat content of a normal rodent chow. Thus, rodent studies with a $60 \%$ fat content might not be as relevant to human physiology as those which use a 40-45\% fat diet (39). Moreover, mice fed with $60 \%$ fat diet become more obese, and do so faster than the ones fed with $40-45 \%$ fat diet. Thus, while many researchers use the $60 \%$ rodent diet as a matter of economics and convenience, it is not the best option for longterm follow-up studies. It is noteworthy that fatty acid (FA) composition of the diet should also be considered besides the percentage of fat in the diet. Moreover, it has been suggested that HFD with high sugar content better mimic the human western diet (40).

For all the aforementioned reasons, we decided to use a customized high fat high sucrose (HFHS) diet containing $40 \%$ energy from animal and vegetal fat (among which $41 \%$ saturated fatty acids, $45 \%$ monounsaturated fatty acids and $14 \%$ polyunsaturated fatty acids) and $25 \%$ by weight sucrose. This diet or its corresponding customized control diet will be given to the mice from 6 to 24 months (Table 1). Interestingly, comparable HFHS diets have been shown to promote sarcopenia, bone loss and impaired neurological function in mice $(41,42)$. These findings represent some of the major features observed in aging humans, suggesting that HFHS diet-fed mice represent a useful model for studying accelerated aging.

\section{Voluntary activity through running wheel access as a model of decelerated aging}

Behavioral paradigms that are commonly used to model human exercise training in mice include forced treadmill running, forced wheel running and voluntary wheel running. Mice running behavior in voluntary wheels is closer to the natural running pattern than forced exercise, as it is performed under non-stress conditions, does not require a negative stimulus, and does not interfere in the normal nocturnal-diurnal rhythmicity of the animal (43). Remarkably, laboratory mice run spontaneously when they have access to running wheels, and this behavior is also observed in feral mice when running wheels are placed in nature (44). Voluntary wheel running thus consists of a rewarding behavior and not a stereotypic behavior that can result from environmental restriction and devoid of any goal or function (45). Another advantage of voluntary wheel running is that, since no direct intervention from the experimenter is required, it can be easily used in long-term studies. Hence, voluntary activity will be assessed in the INSPIRE Mouse cohort by giving mice access to upright running wheels (Table 1 ). To obtain continuous recording throughout the lifespan, we will use a sophisticated method connected to an analysis software that will record detailed activity parameters, including the number and duration of each running period, as well as the number of revolutions, speed, total distance and time, and dark/light cycle activity patterns on running wheels. As mice will be identified by microchips, parameters will be obtained for each single mouse, and at the time of this writing, we are developing a "toll like" detection system to measure individual mouse voluntary activity. Of note, to avoid enrichment/steric hindrance-linked bias, wheels will be placed in all cages. Nevertheless, in the control groups for which the effect of «no physical exercise" will be assessed, running wheels will be blocked (Table 1 ).

\section{Spontaneous mobility}

Mobility is among the most studied and most relevant parameters affecting quality of life with strong prognostic value for disability and survival. Indeed, locomotor impairments in older adults represent a pre-clinical transitional stage towards disability (46). It is thus necessary to understand how agingrelated changes in mobility in mice resemble changes in humans.

To this end, the INSPIRE program will provide a life-long measurement of aging-related locomotor activity in mice, 
THE JOURNAL OF FRAILTY \& AGING

Figure 2

Representation of experimental timeline

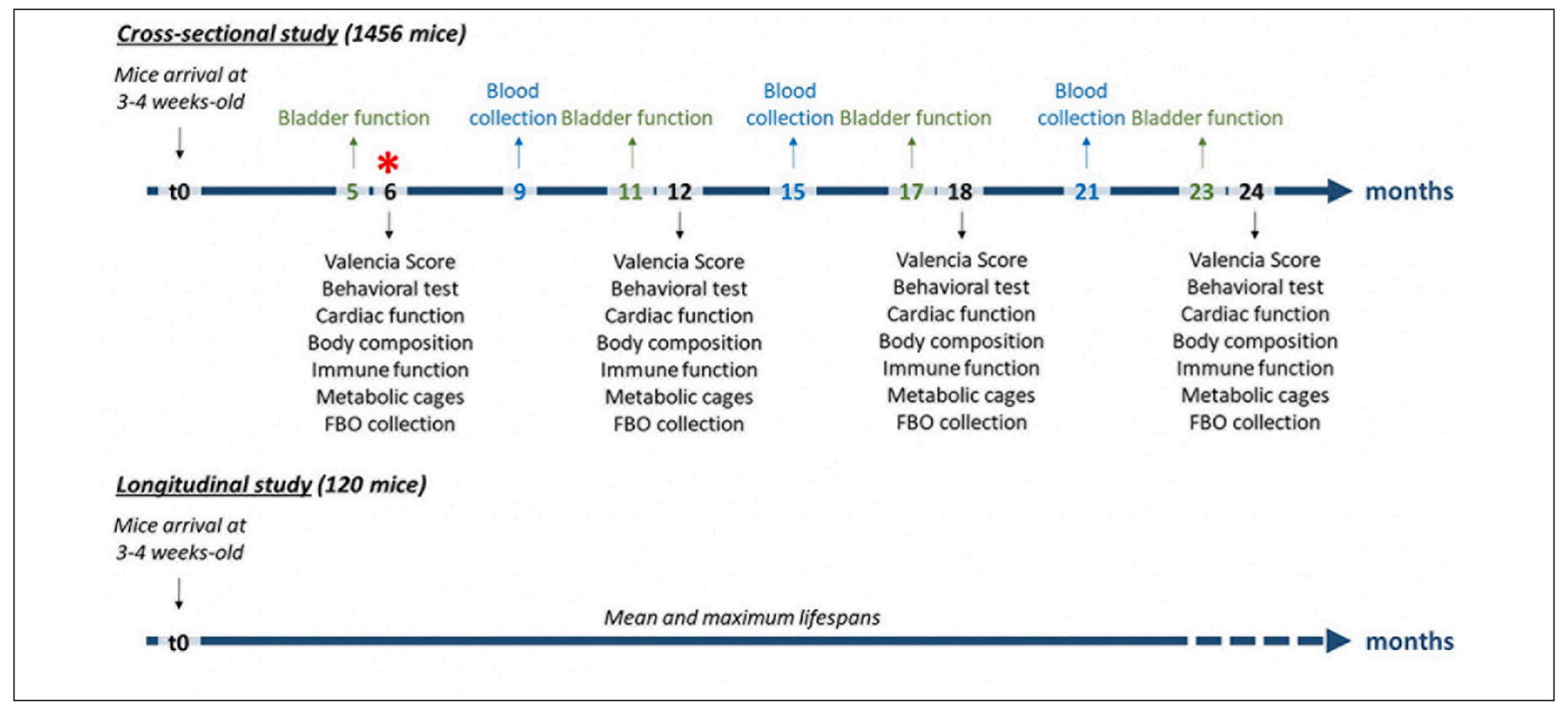

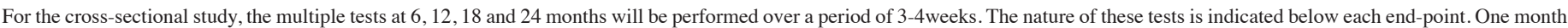

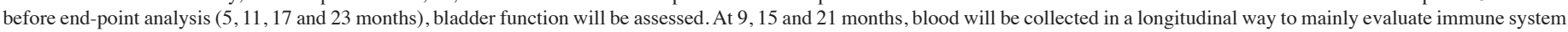

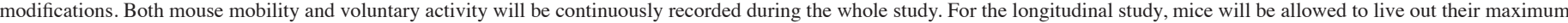
natural lifespan. * indicates the start of the HFHS diet at 6 months. FBO: Feces, Blood, Organs.

through automated home cage monitoring. This technique enables to monitor animals over long periods of time without human intervention. The system we will use, known as Digital Ventilated Cages (DVC®), is designed to gather continuous animal activity data directly from the home cage while keeping cages into conventional Individual Ventilated Cages (IVC) racks (Supp material). It provides a reduction in animal distress thereby increasing welfare, minimization of biases and increased reproducibility of data $(47,48)$. Therefore, mice belonging to the INSPIRE cohort will be housed in DVC cages so locomotor activity of all mice will be continuously and automatically monitored throughout their life. This activity metric represents the overall in-cage activity generated by all mice in a cage from any electrode and is not tracking activity of individual group-housed animals. Therefore, this parameter will be complemented by the individual aforementioned measure, i.e. voluntary activity through running wheel, as well as neuromuscular function by Valencia Score and behavioral cognitive tests (see the following section).

\section{Comprehensive phenotyping}

In this section, we provide an overview of our methodology for the measurement of healthspan and frailty in naturally aging, diet-induced accelerated aging, and exercise-induced decelerated aging in mice. These methods cover a spectrum of highly relevant biological indicators of frailty including cognitive, neuromuscular, cardiac, metabolic and immune function as well as urinary incontinency (For precise timeline, see figure 2). The goal is to improve the currently available "Frailty Scores" with an extended "INSPIRE Frailty Score" suitable for mice, and taking into account accurate parameters to get closer to the human clinical settings $(9,49)$ (Figure 3$)$.

\section{Observational study: longitudinal vs cross-sectional}

Both cross-sectional and longitudinal approaches are observational studies commonly used in aging research. In cross-sectional studies, data are collected as a whole to study a mouse population at a single point in time to examine the relationship between variables of interest. Conversely, in longitudinal studies, data are gathered from the same mouse repeatedly over an extended period of time.

In the case of age-related healthspan studies, data are collected at predetermined ages from multiple individuals within a population. The cross-sectional study design allows performing invasive or terminal procedures but precludes the evaluation of lifespan. In the case of the INSPIRE Mouse cohort, a major cross-sectional study will be conducted with endpoint analyses being performed in different groups of mice at the ages of $6,12,18$ and 24 months-old (Table 1), which roughly correspond to ages from 30 to 70 years in humans. This will allow us to carry out a large number of tests to evaluate and characterize the onset of frailty in aging mice (Figure 2). Importantly, it will also enable to evaluate if some organs "age" prematurely compared to others, and to presume the role of different organ dysfunction in the onset and progression of frailty. 
Conversely, longitudinal studies allow mice to live out their maximum natural lifespan, either dying naturally or being euthanized in case of major decline. Therefore, a longitudinal sub-cohort with 120 animals (60 males and 60 females) will be implemented to the INSPIRE cross-sectional study to evaluate the spontaneous mouse mortality, and to determine mean and maximal lifespans in our animal facilities (Table 1, figure 2).

\section{Frailty evaluation by the "Valencia Score"}

The development of frailty scores suitable for mice and which resemble those that are used in the clinical scenario has become an essential challenge in basic gerontological research. In pursuit of this goal, the "Valencia Score" has been recently developed to measure frailty in rodents (8). It is based on the human clinical parameters described by Linda Fried and co-workers (50), and thus facilitates the extrapolation to humans, as it relies on five robust clinical criteria including unintentional weight loss, weakness, poor endurance, slowness and low activity level, that can be easily measured in mice. According to this score, if a mouse fails three or more components out of five, it is considered as frail, if it fails one or two criteria, it is classified as prefrail, whereas if it does not fail any criteria it is considered as robust, which is equivalent to the clinical classification defined in the Fried Frailty Score. We decided to use the Valencia Score as a starting point to evaluate frailty, and the following parameters will be therefore primarily measured.

\section{Body weight}

Animals' body weights will be recorded biweekly throughout their lifespan to have a precise follow-up of weight evolution. In order to have reliable and individual data, all the mice will be weighted. As suggested by Gomez-Cabrera and colleagues, a 5\% weight loss over a one-month period will be considered positive for this frailty criterion (8), a parameter reflecting the unintentional weight loss commonly observed in frail people.

In order to avoid variability in locomotor activity and other parameters driven by differences in circadian rhythms, all testing will be done starting at the same time. Tests will be run in the order listed, from the least to the most stressful, thereby decreasing the chance that one test might affect the behavior evaluated in the subsequent paradigm.

\section{Grip strength}

The grip strength test is a simple non-invasive method designed to assess neuromuscular function through animal's limb strength. It takes advantage of the animal's tendency to grasp a horizontal metal bar or grid while suspended by its tail. It allows to determine the maximum force, or peak of force, developed by a mouse when the operator tries to move it away from the bar or grid. The measurement is carried out using a high-precision sensor and an electronic device, guaranteeing a perfect capture and display of the maximal force. As suggested by the "Valencia Score", a cut-off point below which $20 \%$ of the observations may be found has to be calculated, and all the animals ranking below this 20th percentile will be considered to fulfill the frailty criterion of weakness, which is frequently measured in the clinical setting.

\section{Motor coordination}

The tightrope test is a method for evaluating neuromuscular coordination and vigor. It is positively correlated to lifespan in rodents and has been extensively validated as a behavioral marker of aging since it was first described in the seventies (51, 52 ). When animals are placed on a tightrope, they are able to grasp the string with the four legs and tail and move to reach a side pole. Mice are scored positive if they are unable to reach the side pole before a $60 \mathrm{sec}$ time-limit or if they fall from the rope. Usually, obese and aged mice cannot lift their hind legs and, after hanging for a few seconds from the forepaws, fall on the cage bedding. In this case, mice are scored as "positive" for this frailty criterion.

\section{Incremental treadmill test}

Poor endurance and slowness are key components of the diagnosis of frailty in humans. These parameters can be evaluated in mice by measuring the running time and speed values when performing an incremental intensity test in a treadmill. For endurance, the running time values will be measured. Then, similar to the grip test, a 20th percentile will be calculated as a cut-off point. The animals that will report a running time under this "threshold" will fulfill this frailty criterion. Besides endurance, running speed will be measured as an index of "slowness". The same aforementioned calculation will be performed to define a threshold under which mice will be considered as positive for the "slowness criterion". Of note, very old animals are usually unable to keep even the lowest running intensities. In our study this is likely to be exacerbated in older mice fed the HFHS diet. As in clinical practice, subjects that are unable to perform any one test are categorized as positive for that criterion.

In the case of the INSPIRE Mouse cohort, the Valencia Score will be used as a primary indicator to evaluate frailty in mice. However, as this score is mainly based on neuromuscular alterations that are commonly observed in frail people, implementation of additional parameters would be of great value to better characterize frailty onset and progression. Therefore, in order to detect early signs of frailty that might not be detected by the Valencia Score, complementary measurements will be carried out on the INSPIRE Mouse cohort in order to propose an extended "INSPIRE Frailty Score", including cognitive, cardiac, metabolic as well as other biological functions (Figure 2). These measurements are described in the following sections. 


\section{THE JOURNAL OF FRAILTY \& AGING}

\section{Behavioral cognitive tests}

Behavioral indicators of healthspan in mice include gait/ ataxia, motivated activity, cognition, and affective function (53). In the context of aging, we will primarily use the spontaneous alternation Y-maze, which assesses prefrontal cortex- and hippocampus-dependent spatial working and reference memory, reflecting changes in cognitive performance (54).

The Y-maze spontaneous alternation test is based on rodent's innate curiosity to explore previously unvisited areas and is used to assess spatial working memory. When placed in a Y-shaped maze, a mouse will show a tendency to enter previously unexplored arms, thus showing alternation in the arm visits. The number of arm entries and the successive entry sequences in the $3 \mathrm{arms}$ are recorded in order to calculate the percentage of alternation. An entry occurs when the four legs are in the arm.

\section{Cardiac function}

Cardiac dysfunction is a main issue in elderly people, and its assessment could be of great interest in the diagnosis and the better characterization of frailty. Despite the absence of underlying pathologies like hypertension or myocardial infarction which lead to heart failure with reduced ejection fraction (HFrEF), the 'normal' aged heart usually exhibits changes like arterial stiffening, increased myocardial stiffness, decreased diastolic myocardial relaxation, increased left ventricular (LV) mass and decreased peak contractility (55). In addition, aging and related comorbidities (obesity, hypertension, diabetes, chronic obstructive disease, anemia and chronic kidney disease) may initiate or aggravate chronic systemic inflammation that may further affect cardiac remodeling and dysfunction (56). Therefore, the majority of elderly patients exhibit heart failure but have a preserved systolic LV function, a syndrome known as heart failure with preserved ejection fraction (HFpEF). Patients with this syndrome have severe symptoms of exercise intolerance, frequent hospitalizations and increased mortality. Despite the importance of $\mathrm{HFpEF}$, optimal treatments remain largely insufficient. The INSPIRE Mouse cohort thus represents a model to better understand HFpEF pathophysiology within a 'systemic' perspective. Of note, approximately $85 \%$ of elderly HFpEF patients are overweight or obese, and the HFpEF epidemic has largely paralleled the obesity epidemic (57). Therefore, HFHS diet-induced obesity also represents a congruent mouse model of HFpEF.

For the evaluation of cardiac function, we have selected echocardiography. In addition to traditional parameters reflecting systolic function (ejection fraction and ventricular wall thickness), particular attention will be given to the measurement of diastolic (dys)function. In particular, the evaluation of mitral inflow will be assessed, as it is very informative and plays an important role in grading diastolic dysfunction (Supp material). Of much interest, these parameters will be complemented with strain imaging to measure the regional and global deformation of the myocardium, which allows for early detection of subclinical LV dysfunction.

The combination of the aforementioned cardiac parameters will allow to better highlight $\mathrm{HFpEF}$ in mice and to upgrade the Valencia Frailty Score with the degree of diastolic dysfunction.

\section{Metabolic function}

During aging, there are changes in body composition, including a loss of lean body mass, bone mass, body water, and a relative increase of fat mass. The bone deteriorates in composition, structure and function, which predisposes to osteoporosis. Furthermore, the increase in fat mass is distributed more specifically in the abdominal region, which is associated with cardiovascular disease and diabetes (58). Changes in body composition often occur in the absence of weight fluctuations, being due to alterations in energy balance, with a positive balance leading to weight gain and a negative balance resulting in weight loss. These key parameters will thus be assessed in the INSPIRE Mouse cohort.

\section{Body composition and bone analysis}

\section{Magnetic Resonance Imaging}

Body composition analysis will be performed by Magnetic Resonance Imaging (MRI) which provides an accurate estimate of whole-body fat, lean, free water, and total water masses in live mice. This technology combines simplicity of use, short scan times, and the comfort of animals which do not need to be anesthetized.

\section{$\underline{X \text {-ray micro computed tomography }}$}

Bone analysis will be done by micro-computed tomography (micro-CT), which can provide ultrahigh-resolution images with resolution of less than $10 \mu \mathrm{m}$. This analysis will be performed after bone collection following terminal anesthesia. This technique will evaluate key parameters of bone microarchitecture like cortical thinning, cortical porosity, thinning of the trabeculae and loss of trabecular connectivity.

\section{Plasmatic metabolic profiling}

In addition to the aforementioned parameters, key plasmatic markers will be measured in plasma collected $2 \mathrm{~h}$ after fasting, at the time of euthanasia. The combination of biochemical and multiplex immunoassay analysis will allow us to determine a broad range of metabolic markers in mice. These markers include, but are not limited to, hepatic enzymes, lipids and lipoproteins, incretins, glycated proteins, glucose, lactic acid, glucagon, insulin, leptin, PYY, amylin, peptide C, ghrelin and others.

The consideration of metabolic function in the INSPIRE frailty score will be of great importance to correlate body and bone compositions, and plasmatic metabolic profiling with neuromuscular and cardiac alterations, which will allow a better 
characterization of the sequential progression of frailty.

\section{Bladder function}

Urinary incontinence is a major problem in the elderly population, especially among women (59). Affected individuals often make great efforts to deny or hide urinary incontinence, which can lead to psychosocial hindrance. Its consideration is thus important in the characterization of frailty, but unfortunately its measurement is often undervalued in aging research, in particular in animal cohorts.

We thus decided to measure urinary incontinence in the INSPIRE Mouse cohort in order to study lower urinary tract function during aging. To this end, a spontaneous void spot assay (VSA) will be performed (Supp material), so urinary spotting patterns will be used as an indirect way of measuring bladder function and outlet control (60). As urinary incontinence is usually considered as a feature of frailty in humans, its measurement in mice will improve the scoring of frailty to be closer to the clinical evaluation.

\section{Immune function}

A crucial component of aging is a set of alterations in the immune system that can manifest as a decreased ability to fight infection, diminished response to vaccination, increased incidence of cancer and constitutive low-grade inflammation (61). The latter, which has been called "inflammaging", has drawn particular attention in the field of aging, as recent studies have provided evidence that a pool of molecules can be secreted by senescent cells, a process known as senescence-associated secretory phenotype (SASP). This SASP includes cytokines, chemokines, proteases and growth factors that can affect neighboring cells via autocrine/paracrine pathways.

Immunological markers will be assessed in the INSPIRE Mouse cohort at different time points, i.e. 9, 15 and 21 months through submandibular blood collection and 6, 12, 18 and 24 months through terminal blood collection in the posterior vena cava (Figure 2). These markers will be measured in plasma by multiplex immunoassays and include, but are not limited to, IL6, IL-1 beta, TNF alpha, IL-12, IFN gamma, IL-2, IL-10, TGF beta, IL-4, IL-13, IL-17, CCL2, CXCL9, CXCL10, CCL22, CCL17, CRP. In addition, end-point blood collection will also serve at determining the white blood cell count of mice.

Adding some key markers reflecting immune system modifications in the characterization of frailty would be of great interest, as this feature is not considered in the current evaluation of frailty in mice.

\section{Organ collection, biobanking and multi-omics analysis}

After phenotyping, mice will be sacrificed and urine, feces, blood and tissues will be collected for biobanking as appropriate. Mice will be fasted $2 \mathrm{~h}$ before euthanasia. Urine will be collected after placing mice in metabolic cages for 12 hours, the day before euthanasia. Feces will be collected during mouse handling, just before euthanasia and directly frozen. Blood will be collected just after euthanasia from the posterior vena cava, which is recommended for terminal stage studies in order to collect a maximal volume of blood. The fluids will be prepared as appropriate (e.g. for plasma collection), aliquoted and stored at $-80^{\circ} \mathrm{C}$ before further investigations. Concerning the tissues, as many tissues as possible will be collected. Each tissue will be then subdivided into two pieces: the first one will be included in Optimal Cutting Temperature (OCT) compound, paraformaldehyde (PFA) or glutaraldehyde as appropriate, and cut into ultrathin slices for complete anatomopathological analysis. The other piece will be flash frozen in liquid nitrogen and, shortly before analysis, tissues will be fragmented with a biopulverizer into tiny pieces the size of grains of sand or course powder. This technique was selected for different reasons: 1) it reduces the number of collector tubes; 2) it limits sampling bias during organ collection; and 3) it optimizes subsequent rapid and complete lysis using lytic solutions or mechanical homogenizers. All the samples will be stored in a Biological Resource Center dedicated to the conservation of biological resources according to strict criteria of ethics and quality.

Multi-Omics analysis will be performed on biological fluids, feces and tissues. To facilitate the transfer of the results to the Human cohort, priority will be given to the analyses in plasma, urine (in particular proteomics and metabolomics profiling) and feces (microbiota analysis). The goal of this approach is to define a set of robust and accurate biomarkers for normal, accelerated, and decelerated aging. The tissues will be then dedicated to the multi-Omics-designed identification of novel tissue-specific candidate biomarkers for frailty and accelerated/decelerated aging, and to the validation of the novel candidate targets for prevention and treatment of accelerated aging (Figure 3).

\section{Conclusions and perspectives}

Belonging to the global INSPIRE platform on geroscience, the INSPIRE Mouse cohort represents a unique way to model and better characterize frailty in mice. Although excellent institutions like the Buck Institute and the National Institute on Aging also carry out comparable studies in mice dedicated to investigate biological aging, the main originality of the INSPIRE Mouse cohort relies on the focus on getting closer to the human lifestyle to define the time course and the mechanisms of frailty/accelerated aging onset. Within this line, the selection of outbred mice that better parallel human genetic diversity, is a determining parameter offering more generalizability of responses across populations. In addition, including both males and females, and mimicking "humanized" lifestyles through voluntary physical activity and HFHS-diet induced obesity further approach real human living conditions.

Through a large functional and biological phenotyping of 
Figure 3

Graphical abstract of the proposed INSPIRE Frailty Score

\section{« INSPIRE FRAILTY SCORE »}

\section{FUNCTIONAL FRAILTY}

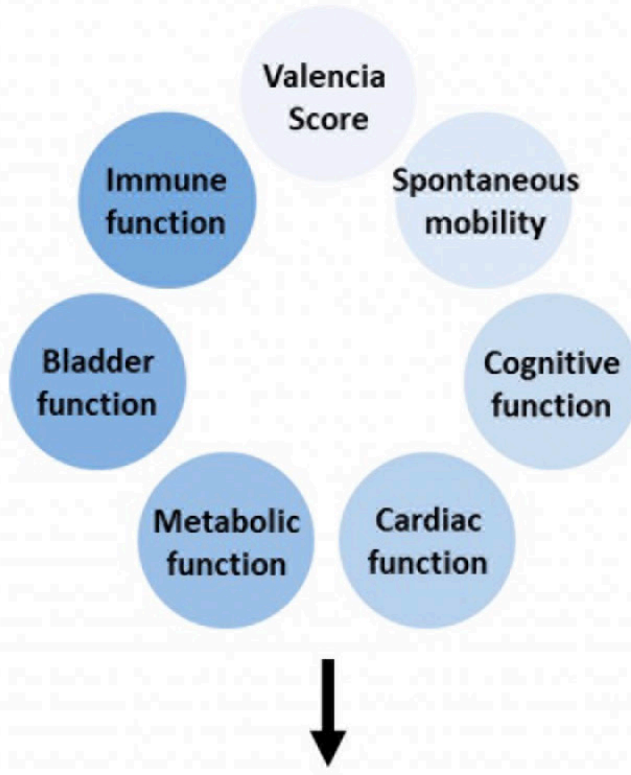

Clinically RELEVANT

DATASET

\section{BIOLOGICAL FRAILTY}

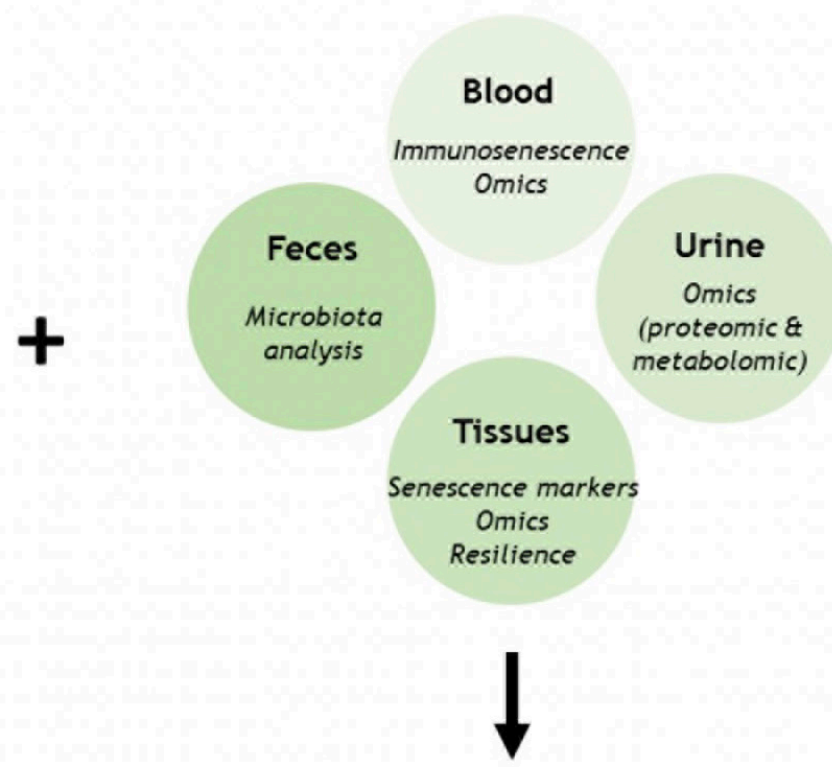

FRAILTY

BIOMARKERS

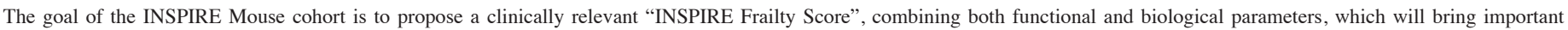
knowledge on frailty characterization, assessment and target identification.

mice, a first objective of the INSPIRE project is to define the age at which early signs of frailty arise. Indeed, frailty is considered as a clinical syndrome appearing in advanced ages (62), but this is because the definition of frailty is mainly based on clinical criteria becoming discriminating in old patients. However, it is likely that the biological mechanisms leading to frailty and accelerated aging may be induced and detectable much earlier than the actual clinical signs of frailty. The goal here is to define the early signs of premature aging and to correlate them to the normal/altered functional phenotype to $1 /$ define the age of frailty onset and 2/ identify the organ/ system(s) primarily altered in the frailty process. To this aim, the development of a clinically relevant score for frailty in mice is essential. Within this line, the "Howlett and Rockwood frailty index" is a simple and noninvasive index, based on 31 health-related variables like alopecia, distended abdomen, hearing loss and breathing rate (9). Although this 31-item check list is based on deficit accumulation during aging, we believe that investigator bias may play a critical role in diagnosis of frailty, which may affect the comparison of results across studies. More recently, the Valencia Score has been developed to determine frailty in naturally aging mice, based on five clinical components previously reported for humans by Fried and co-workers $(8,50)$. Despite its undeniable interest, this approach is primarily focused on the in-depth study of agingrelated neuromuscular alterations and does not evaluate other key aspects of frailty such as cognitive, cardiac or metabolic impairments. Therefore, for the INSPIRE Mouse cohort, mice will be initially labeled as 'frail/pre-frail/robust' based solely on the Valencia test. Then, functional phenotyping will allow us to know if other aspects of frailty that are currently undervalued (e.g. cardiac or metabolic alterations) are detectable earlier than neuromuscular defects, which could greatly refine frailty detection. Then, a cut-off will be empirically determined for each parameter in order to set a more accurate frailty score. This method will bring key information on frailty by $1 /$ evaluating the effect of HFHS-induced overweight and sedentarism on frailty onset and 2 / including clinically relevant criteria like cognitive, cardiac, metabolic, bladder and immune parameters in addition to the currently measured neuromuscular 
deficits (Figure 3). Importantly, all these parameters, which will be supplemented by the longitudinal follow up of mouse mobility and voluntary activity, closely reflect changes observed in humans and therefore better approach the human frailty criteria.

Besides phenotypic measures, molecular biomarkers will be highly valuable and complementary in the prediction of healthy/unhealthy aging. Through a better understanding of the close relationship between the molecular mechanisms of cell premature aging and the onset of frailty/accelerated aging, the INSPIRE Mouse cohort will foster the identification of a panel of robust and sensitive frailty biomarkers that have not been extensively studied so far. Multi-Omics analysis of blood, urine and feces will allow to rapidly identify such biomarkers' profiles (that can be conceptualized as a "frailty ID"), which might inform timely pharmacological and non-pharmacological preventive strategies acting directly on aging and contributing to a healthy state even in late ages. Then, these multi-Omics approaches will be extended to tissues to eventually discover novel tissue-specific putative biomarkers and therapeutic targets of frailty/accelerated aging (Figure 3).

An important notion, tightly linked to frailty is resilience, which is defined as the capacity to respond to or recover from clinically relevant stresses (63). Therefore, resilience must be evaluated in aging studies and necessitates the development of new animal models, which would be of particular great value for testing the benefits of geroprotectors. However, modelling resilience in mice is challenging, as there is no consensus on its precise definition or on how best to measure it (64). Although some models are currently available, there is very little data related to the characterization of the multiple deficits caused, especially in aged animals. As of this writing, INSPIRE investigators (gathering physicians, pharmacists, epidemiologists, geriatricians, clinicians, molecular biologists and others interested in the process of aging) are working on the tremendous question of "resilience modelling", aiming at reaching a consensus on the suitability of such models.

To sum up, the INSPIRE Mouse cohort will importantly lead to the precise functional characterization of frailty together with the identification of robust molecular biomarkers to predict healthy/unhealthy aging. The resulting INSPIRE Frailty Score, combining both functional and biological parameters, will thus allow to refine frailty characterization and detection in animal models (figure 3 ). Therefore, by belonging to the global INSPIRE platform on geroscience $(6,65)$ and through its interaction with the INSPIRE Human Translational cohort and the INSPIRE Icope Care Cohort $(6,7,66)$, the INSPIRE Mouse cohort should speed up the discovery process in the field of aging, with the final goal to increase access to healthy aging for the current and next generations.

Acknowledgments: We thank Massimiliano Bardotti, Rémy Burcelin and Sarah Gandarillas for their help in the design of the cohort. The Inspire Program was supported by grants from the Region Occitanie/Pyrénées-Méditerranée (Reference number: 1901175), the European Regional Development Fund (ERDF) (Project number: MP0022856), and the Inspire Chairs of Excellence funded by: Alzheimer Prevention in Occitania and Catalonia (APOC), EDENIS, KORIAN, Pfizer, Pierre-Fabre.

Conflict of interest: All authors of the paper "Towards a large-scale assessment of relationship between biological and chronological aging: The INSPIRE Mouse cohort" declare no conflict of interest related to this manuscript.

Permissions: This article is distributed under the terms of the Creative Commons Attribution 4.0 International License (http://creativecommons.org/licenses/by/4.0/), which permits use, duplication, adaptation, distribution and reproduction in any medium or format, as long as you give appropriate credit to the original author(s) and the source, provide a link to the Creative Commons license and indicate if changes were made.

Open Access: This article is distributed under the terms of the Creative Commons Attribution 4.0 International License (http://creativecommons.org/licenses/by/4.0/), which permits use, duplication, adaptation, distribution and reproduction in any medium or format, as long as you give appropriate credit to the original author(s) and the source, provide a link to the Creative Commons license and indicate if changes were made.

\section{References}

1. Niccoli T, Partridge L. Ageing as a Risk Factor for Disease. Current Biology 2012;22:R741-R752

2. Kennedy BK, Berger SL, Brunet A, et al. Geroscience: linking aging to chronic disease. Cell 2014;159:709-713

3. Sierra F. The Emergence of Geroscience as an Interdisciplinary Approach to the Enhancement of Health Span and Life Span. Cold Spring Harb Perspect Med 2016;6

4. Cesari M, Calvani R, Marzetti E. Frailty in Older Persons. Clinics in Geriatric Medicine 2017;33:293-303

5. Wallace RG, Twomey LC, Custaud M-A, et al. Potential Diagnostic and Prognostic Biomarkers of Epigenetic Drift within the Cardiovascular Compartment. BioMed Research International. https://www.hindawi.com/journals/bmri/2016/2465763/. Accessed 28 May 2020

6. de Souto Barreto P, Guyonnet S, Ader I, et al. The Inspire Research Initiative: A Program for Geroscience and Healthy Aging Research going from Animal Models to Humans and the Healthcare System. J Frailty Aging 2020

7. Takeda C, Guyonnet S, Sumi Y, Vellas B, Araujo de Carvalho I. Integrated Care for Older People and the Implementation in the INSPIRE Care Cohort. J Prev Alzheimers Dis 2020;7:70-74

8. Gomez-Cabrera MC, Garcia-Valles R, Rodriguez-Mañas L, et al. A New Frailty Score for Experimental Animals Based on the Clinical Phenotype: Inactivity as a Model of Frailty. J Gerontol A Biol Sci Med Sci 2017;72:885-891

9. Whitehead JC, Hildebrand BA, Sun M, et al. A Clinical Frailty Index in Aging Mice: Comparisons With Frailty Index Data in Humans. J Gerontol A Biol Sci Med Sci 2014;69:621-632

10. Towards frailty biomarkers: Candidates from genes and pathways regulated in aging and age-related diseases. Ageing Research Reviews 2018;47:214-277

11. Herr M, Cesari M, Landre B, et al. Factors associated with changes of the frailty status after age 70: Findings in the MAPT study. Ann Epidemiol 2019;34:65-70.e1

12. Landré B, Czernichow S, Goldberg M, Zins M, Ankri J, Herr M. Association Between Life-Course Obesity and Frailty in Older Adults: Findings in the GAZEL Cohort. Obesity 2020;28:388-396

13. da Silva VD, Tribess S, Meneguci J, et al. Association between frailty and the combination of physical activity level and sedentary behavior in older adults. BMC Public Health 2019;19:709

14. Kehler DS, Hay JL, Stammers AN, et al. A systematic review of the association between sedentary behaviors with frailty. Exp Gerontol 2018;114:1-12

15. Miller AA, Spencer SJ. Obesity and neuroinflammation: a pathway to cognitive impairment. Brain Behav Immun 2014;42:10-21

16. Yeomans MR. Adverse effects of consuming high fat-sugar diets on cognition: implications for understanding obesity. Proc Nutr Soc 2017;76:455-465

17. Chung ST, Onuzuruike AU, Magge SN. Cardiometabolic risk in obese children. Ann N Y Acad Sci 2018;1411:166-183

18. Aronne LJ, Isoldi KK. Overweight and obesity: key components of cardiometabolic 


\section{THE JOURNAL OF FRAILTY \& AGING}

risk. Clin Cornerstone 2007:8:29-37

19. Andersen CJ, Murphy KE, Fernandez ML. Impact of Obesity and Metabolic Syndrome on Immunity 12. Adv Nutr 2016;7:66-75

20. Tamura Y, Ishikawa J, Fujiwara Y, et al. Prevalence of frailty, cognitive impairment, and sarcopenia in outpatients with cardiometabolic disease in a frailty clinic. BMC Geriatr 2018;18

21. Hwang A-C, Liu L-K, Lee W-J, et al. Association of Frailty and Cardiometabolic Risk Among Community-Dwelling Middle-Aged and Older People: Results from the I-Lan Longitudinal Aging Study. Rejuvenation Res 2015;18:564-572

22. Yuan R, Peters LL, Paigen B. Mice as a mammalian model for research on the genetics of aging. ILAR J 2011;52:4-15

23. Johnson M. Laboratory Mice and Rats. Materials and Methods 2020

24. Festing MF. Warning: the use of heterogeneous mice may seriously damage your research. Neurobiol Aging 1999;20:237-244; discussion 245-246

25. Jensen VS, Porsgaard T, Lykkesfeldt J, Hvid H. Rodent model choice has major impact on variability of standard preclinical readouts associated with diabetes and obesity research. Am J Transl Res 2016;8:3574-3584

26. Tuttle AH, Philip VM, Chesler EJ, Mogil JS. Comparing phenotypic variation between inbred and outbred mice. Nat Methods 2018;15:994-996

27. Hsieh LS, Wen JH, Miyares L, Lombroso PJ, Bordey A. Outbred CD1 mice are as suitable as inbred $\mathrm{C} 57 \mathrm{BL} / 6 \mathrm{~J}$ mice in performing social tasks. Neurosci Lett 2017;637:142-147

28. Aldinger KA, Sokoloff G, Rosenberg DM, Palmer AA, Millen KJ. Genetic Variation and Population Substructure in Outbred CD-1 Mice: Implications for Genome-Wide Association Studies. PLoS One 2009;4

29. Gao M, Ma Y, Liu D. High-fat diet-induced adiposity, adipose inflammation, hepatic steatosis and hyperinsulinemia in outbred CD-1 mice. PLoS ONE 2015;10:e0119784

30. Buttigieg A, Flores O, Hernández A, Sáez-Briones P, Burgos H, Morgan C. Preference for high-fat diet is developed by young Swiss CD1 mice after short-term feeding and is prevented by NMDA receptor antagonists. Neurobiol Learn Mem 2014;107:13-18

31. Chen K, Zheng X, Feng M, Li D, Zhang H. Gut Microbiota-Dependent Metabolite Trimethylamine N-Oxide Contributes to Cardiac Dysfunction in Western Diet-Induced Obese Mice. Front Physiol 2017;8:139

32. Zhang H, Meng J, Yu H. Trimethylamine N-oxide Supplementation Abolishes the Cardioprotective Effects of Voluntary Exercise in Mice Fed a Western Diet. Front Physiol 2017;8

33. Anderson NJ, King MR, Delbruck L, Jolivalt CG. Role of insulin signaling impairment, adiponectin and dyslipidemia in peripheral and central neuropathy in mice. Dis Model Mech 2014;7:625-633

34. Ramos-Ibeas P, Pericuesta E, Peral-Sanchez I, et al. Longitudinal analysis of somatic and germ-cell telomere dynamics in outbred mice. Mol Reprod Dev 2019;86:10331043

35. Antoch MP, Wrobel M, Kuropatwinski KK, et al. Physiological frailty index (PFI) quantitative in-life estimate of individual biological age in mice. Aging (Albany NY) 2017;9:615-626

36. Clayton JA, Collins FS. Policy: NIH to balance sex in cell and animal studies. Nature News 2014;509:282

37. Pettersson US, Waldén TB, Carlsson P-O, Jansson L, Phillipson M. Female Mice are Protected against High-Fat Diet Induced Metabolic Syndrome and Increase the Regulatory T Cell Population in Adipose Tissue. PLoS ONE 2012;7:e46057

38. Porter Starr KN, McDonald SR, Bales CW. Obesity and Physical Frailty in Older Adults: A Scoping Review of Intervention Trials. J Am Med Dir Assoc 2014;15:240250

39. Speakman JR. Use of high-fat diets to study rodent obesity as a model of human obesity. Int J Obes 2019;43:1491-1492

40. Heydemann A. An Overview of Murine High Fat Diet as a Model for Type 2 Diabetes Mellitus. Journal of Diabetes Research. https://www.hindawi.com/journals/ jdr/2016/2902351/. Accessed 8 Apr 2020

41. Burchfield JG, Kebede MA, Meoli CC, et al. High dietary fat and sucrose result in an extensive and time-dependent deterioration in health of multiple physiological systems in mice. J Biol Chem 2018;293:5731-5745
42. Rasool S, Geetha T, Broderick TL, Babu JR. High Fat With High Sucrose Diet Leads to Obesity and Induces Myodegeneration. Front Physiol 2018;9

43. Goh J, Ladiges W. Voluntary Wheel Running in Mice. Current Protocols in Mouse Biology 2015;5:283-290

44. Meijer JH, Robbers Y. Wheel running in the wild. Proceedings of the Royal Society B: Biological Sciences 2014;281:20140210

45. Manzanares G, Brito-da-Silva G, Gandra PG, Manzanares G, Brito-da-Silva G, Gandra PG. Voluntary wheel running: patterns and physiological effects in mice. Brazilian Journal of Medical and Biological Research 2019;52

46. Ferrucci L, Cooper R, Shardell M, Simonsick EM, Schrack JA, Kuh D. Age-Related Change in Mobility: Perspectives From Life Course Epidemiology and Geroscience. J Gerontol A Biol Sci Med Sci 2016;71:1184-1194

47. Iannello F. Non-intrusive high throughput automated data collection from the home cage. Heliyon 2019;5:e01454

48. Pernold K, Iannello F, Low BE, et al. Towards large scale automated cage monitoring - Diurnal rhythm and impact of interventions on in-cage activity of C57BL/6J mice recorded 24/7 with a non-disrupting capacitive-based technique. PLOS ONE 2019;14:e0211063

49. Martinez de Toda I, Garrido A, Vida C, Gomez-Cabrera MC, Viña J, De la Fuente M. Frailty Quantified by the "Valencia Score" as a Potential Predictor of Lifespan in Mice. J Gerontol A Biol Sci Med Sci 2018;73:1323-1329

50. Fried LP, Tangen CM, Walston J, et al. Frailty in Older AdultsEvidence for a Phenotype. J Gerontol A Biol Sci Med Sci 2001;56:M146-M157

51. Miquel J, Blasco M. A simple technique for evaluation of vitality loss in aging mice, by testing their muscular coordination and vigor. Experimental Gerontology 1978;13:389-396

52. Matheu A, Maraver A, Klatt $\mathrm{P}$, et al. Delayed ageing through damage protection by the Arf/p53 pathway. Nature 2007;448:375-379

53. Ackert-Bicknell CL, Anderson L, Sheehan S, et al. Aging Research Using Mouse Models. Curr Protoc Mouse Biol 2015;5:95-133

54. Kraeuter A-K, Guest PC, Sarnyai Z. The Y-Maze for Assessment of Spatial Working and Reference Memory in Mice. Methods Mol Biol 2019;1916:105-111

55. Strait JB, Lakatta EG. Aging-associated cardiovascular changes and their relationship to heart failure. Heart Fail Clin 2012;8:143-164

56. Upadhya B, Kitzman DW. Heart Failure With Preserved Ejection Fraction in Older Adult. Heart Fail Clin 2017;13:485-502

57. Ndumele CE, Coresh J, Lazo M, et al. Obesity, subclinical myocardial injury, and incident heart failure. JACC Heart Fail 2014;2:600-607

58. St-Onge M-P, Gallagher D. Body composition changes with aging: The cause or the result of alterations in metabolic rate and macronutrient oxidation? Nutrition 2010;26:152-155

59. Vogel SL. Urinary Incontinence in the Elderly. Ochsner J 2001;3:214-218

60. Hill WG, Zeidel ML, Bjorling DE, Vezina CM. Void spot assay: recommendations on the use of a simple micturition assay for mice. Am J Physiol Renal Physiol 2018;315:F1422-F1429

61. Sadighi Akha AA. Aging and the immune system: An overview. J Immunol Methods 2018;463:21-26

62. Vellas B, Sourdet S. Prevention of Frailty in Aging. J Frailty Aging 2017;6:174-177

63. Kirkland JL, Stout MB, Sierra F. Resilience in Aging Mice. J Gerontol A Biol Sci Med Sci 2016;71:1407-1414

64. Schosserer M, Banks G, Dogan S, et al. Modelling physical resilience in ageing mice Mech Ageing Dev 2019;177:91-102

65. Guerville F, De Souto Barreto P, Ader I, et al. Revisiting the Hallmarks of Aging to Identify Markers of Biological Age. J Prev Alzheimers Dis 2020;7:56-64

66. Beard JR. Linking geroscience and integrated care to reinforce prevention. Journal of Prevention of Alzheimer's Disease 2020;J Prev Alz Dis 20207:68-69 